\title{
Revisión sistemática de literatura sobre factores clave en la identificación de oportunidades de negocio
}

\section{Systematic literature review about key factors in the identification of business opportunities}

\begin{abstract}
Mg. Harold Gamero profesor de la Universidad Católica San Pablo, Perú, es Maestro en Investigación en Ciencias de la Administración por la Universidad Esan. (h.gamero@outlook.com) (orcid.org/0000-0001-8614-3966)
\end{abstract}

Dr. Jhony Ostos profesor de la Universidad Esan, es doctor en Management Science por la Universidad Ramon Llull - ESADE, España. (jostos@esan.edu.pe) (orcid.org/0000-0003-1888-7378)

\begin{abstract}
Resumen
La identificación de oportunidades de negocio es una tarea determinante dentro de la dinámica del emprendimiento. Por ello, diferentes investigaciones han buscado relaciones entre los factores individuales y los resultados exitosos de este primer paso emprendedor. Aunque numerosos factores han sido analizados existe todavía una extensión considerable de definiciones que varían entre ellos. Atendiendo a esta necesidad, este artículo desarrolla una revisión sistemática de la literatura, consolidando aquellos factores que han sido incluidos en investigaciones de este campo durante los últimos diez años. Esta revisión se llevó a cabo siguiendo la metodología trietápica de Jennifer Platt, la cual ha sido ampliamente utilizada en artículos similares. Partiendo de un total de 3119 artículos, se hizo una depuración sistemática teniendo como resultado final un análisis de 30 artículos. Los resultados de este estudio indican que existen alrededor de 22 factores propuestos, siendo cinco los factores más importantes que facilitan la identificación de oportunidades de negocio: el conocimiento previo, el estado de alerta emprendedor, la autoeficacia, la creatividad, y las redes de contacto. Otros factores encontrados en el análisis son: la experiencia en emprendimiento, la experiencia laboral, la educación, las capacidades cognitivas, las intenciones de emprendimiento y la búsqueda activa de oportunidades en el mercado. Este trabajo contribuye a la literatura resumiendo los factores incluidos en múltiples investigaciones y ofreciendo líneas futuras de investigación para académicos interesados en el área.
\end{abstract}

\begin{abstract}
The identification of business opportunities is a determining task within the dynamics of entrepreneurship. Therefore, different researches have sought relationships between individual factors and the successful results of this first entrepreneurial step. Although numerous factors have been analyzed, there is still a considerable extension of definitions that vary between them. In response to this need, this article develops a systematic review of the literature, consolidating those factors that have been included in this research field during the last ten years. This review was carried out following the Jennifer Platt three-stage methodology, which has been widely used in similar articles. Starting from a total of 3119 articles, a systematic depuration was carried out resulting in an analysis of 30 articles. The results of this study indicate that there are about 22 proposed factors, with five being the most important among them, that facilitate the identification of business opportunities: prior knowledge, entrepreneurial alertness, self-efficacy, creativity, and contact networks. Other factors found in the analysis are: entrepreneurship experience, work experience, education, cognitive abilities, entrepreneurship intentions and the active search for opportunities in the market. This work contributes to the literature by summarizing the factors included in multiple investigations and offering future lines of research for academics interested in the area.
\end{abstract}

\section{Palabras clave I keywords}

Emprendimiento, identificación de oportunidades, conocimiento previo, experiencia de emprendimiento, estado de alerta emprendedor, autoeficacia, creatividad, redes de contacto.

Entrepreneurship, opportunity identification, prior knowledge, entrepreneurship experience, entrepreneurial alertness, self-efficacy, creativity, social networks.

Cómo citar: Gamero, H., y Ostos, J. (2020). Revisión sistemática de literatura sobre factores clave en la identificación de oportunidades de negocio. Retos Revista de Ciencias de la Administración y Economía, 10(20), pp. 307-327. https://doi.org/10.17163/ret.n20.2020.07 


\section{Introducción}

Las oportunidades de negocio son el elemento central sobre el cual se desarrolla el proceso del emprendimiento. Desde teorías iniciales, como la de Schumpeter (1934), hasta los modelos sobre emprendimiento vigentes, como los planteados por Venkataraman (1997), Shane y Venkataraman (2000) o Eckhardt y Shane (2003), estas oportunidades se definen como circunstancias que favorecen a las actividades de emprendimiento (George et al., 2016). Más formalmente, las oportunidades de negocio son aquellas situaciones en las cuales nuevos productos, servicios, materias primas o métodos de organización pueden ser introducidos en un mercado y vendidos a un precio superior a los costos de producción (Shane \& Venkataraman, 2000). Sin embargo, los emprendedores no son únicamente comerciantes que compran y venden recursos en diferentes momentos del tiempo y a diferentes valores, sino individuos capaces de recombinar los recursos que encuentran disponibles (Shane, 2012). De esta manera, el emprendimiento planteado por Shane y Venkataraman (2000) es un proceso de tres etapas que consiste en la identificación, evaluación y explotación de oportunidades de negocio.

Una de las preguntas que más ha llamado el interés de investigadores del área, es el por qué algunas personas son capaces de identificar oportunidades de negocio mientras otras no (Baron, 2006)suggested by research on human cognition, is that they do so by using cognitive frameworks they have acquired through experience to perceive connections between seemingly unrelated events or trends in the external world. In other words, they use cognitive frameworks they possess to connect the dots between changes in technology, demographics, markets, government policies, and other factors. The patterns they then perceive in these events or trends suggest ideas for new products or servicesideas that can potentially serve as the basis for new ventures. This pattern recognition perspective on opportunity identification is useful in several respects. First, it helps integrate into one basic framework three factors that have been found to play an important role in opportunity recognition: engaging in an active search for opportunities; alertness to them; and prior knowledge of an industry or market. In addition, it also helps explain interrelations between these factors (e.g., the fact that active search may not be required when alertness is very high, la cual se centra en el primer paso de este proceso emprendedor. Diversos estudios coinciden en que existen diferencias cognitivas, psicologías y emocionales entre los emprendedores, las cuales condicionan y moderan la capacidad de una persona para reconocer oportunidades de negocio rentables (Gartner et al., 2008). A partir de estas afirmaciones, otras investigaciones se han enfocado en identificar y estudiar los factores que potencian la identificación de oportunidades (Grégoire et al., 2011), sin embargo, la proliferación de estas investigaciones hace necesario el análisis de la amplitud y variedad de los conceptos que en ellas se incluyen.

El presente artículo tiene como objetivo desarrollar una revisión sistemática de la literatura sobre los factores individuales relevantes para la identificación de oportunidades de negocio. Para Webster y Watson (2011) las revisiones sistemáticas son insumos de gran valor que generan una base sólida para el desarrollo del conocimiento posterior: facilitan el desarrollo de teorías, ayuda a concluir en áreas del concomimiento con contenido excesivo e innecesario o, por el contrario, descubren áreas donde es necesaria mayor investigación. 
Este artículo se presenta de la siguiente manera. Primero, se detalla la metodología empleada para la búsqueda, selección y análisis de las publicaciones relevantes, incluidas para esta revisión de la literatura. Segundo, se listan y explican los factores individuales con clasificación de competencias. En las últimas dos secciones se resumen los hallazgos más importantes encontrados en este estudio y se sugieren investigaciones futuras en función a las brechas del conocimiento encontradas.

\section{Metodología para la revisión de la literatura}

Los parámetros utilizados para esta revisión se han extraído de prácticas utilizadas en otros análisis similares previos, tales como son las revisiones sistemáticas de Fayezi et al. (2017), Hmieleski y Powell (2017), George et al. (2016) y Mitchelmore y Rowley (2013); los meta-análisis desarrollados por Bae et al. (2014), Martin et al. (2013) y Miao et al. (2017); y las recomendaciones planteadas por Sánchez-Meca (2010) y Webster y Watson (2011). Con base en estos trabajos se han tomado en consideración los siguientes parámetros: artículos empíricos evaluados por pares expertos, publicados en revistas científicas de la especialidad durante los últimos 10 años (enero 2009-abril 2019), en idioma inglés y español. Las bases de datos utilizadas para la búsqueda fueron: ABI/Inform, Emerald y Web of SCience ya que estas incluyen investigaciones importantes sobre oportunidades de emprendimiento (George et al., 2016).

La búsqueda de fuentes bibliográficas se llevó a cabo siguiendo la metodología propuesta por Platt (1981), la cual ha sido utilizada en revisiones previas (Fayezi et al., 2017). Esta metodología propone tres etapas que ayudan a identificar y evaluar publicaciones relevantes sobre el tema, a través de un proceso de depuración sistemática.

En la primera etapa, se realizó la búsqueda haciendo uso de las palabras clave: entrepreneurship, opportunity recognition, opportunity discovery, opportunity creation, opportunity identification, atendiendo a las tres posibles perspectivas de identificación de oportunidades planteadas por Sarasvathy et al. (2003). De esta búsqueda se obtuvieron 3,119 artículos. De este total se separaron aquellos artículos no pertenecientes al campo del emprendimiento, así como editoriales, capítulos y revisiones de libro, noticias, documentos duplicados y otros con características diferentes a las de interés, tras lo cual se mantuvieron 2495 artículos.

En la segunda etapa, se realizó un análisis de términos en títulos, resúmenes y palabras clave (Fayezi et al., 2017) a fin de identificar aquellos que no coincidían con las intenciones de esta revisión. Para ello se eligieron únicamente aquellos documentos que presentaban un enfoque hacia el emprendimiento como un proceso centrado en las oportunidades y que, además, se enfocaran en la primera etapa del modelo planteado por Shane y Venkataraman (2000), resultando en 475 artículos depurados. De este total, se extrajeron 15 revisiones de literatura, dos meta-análisis, dos artículos metodológicos, y 47 estudios de caso.

En la tercera etapa, se revisaron a profundidad los resúmenes y conclusiones de los artículos resultantes y se excluyeron aquellos cuyas unidades de análisis no eran individuos o que no consideraban uno o más rasgos o factores de la personalidad. Al final de este proceso se obtuvieron 30 artículos: 1 con la aproximación de creación de oportunidades, 4 con la aproximación de descubrimiento, 11 con la de reconocimiento y 14 con la aproximación de identificación de oportunidades de emprendimiento. 
Este proceso se llevó a cabo de forma manual y con el apoyo del programa de hojas de cálculo Excel. Algunos de los campos incluidos en la tabla elaborada para el análisis fueron: perspectiva sobre la oportunidad, autor(es), títulos, año, revista, intención del documento, conclusiones, entre otros. En la sección siguiente se muestran los hallazgos más importantes encontrados.

\section{Hallazgos principales}

En la Tabla 1 se resumen los resultados más importantes encontrados en cada uno de los artículos incluidos en esta revisión. En la Tabla 2 se listan los factores individuales relevantes para la identificación de oportunidades de negocio, según los artículos en los cuales han sido incluidos. El Gráfico 1 es una representación visual de la frecuencia en la que los factores han sido incluido en los artículos analizados, a través de una nube de palabras. Las tablas 1 y 2 están ordenadas cronológicamente, iniciando con el artículo de mayor antigüedad y terminando con el más reciente.

Para agrupar las características encontradas se tomó como base la clasificación de competencias individuales sugerida por Lahti (1999), donde se las define como un conjunto interrelacionado de conocimientos, capacidades, habilidades, y otras características que contribuyen al éxito de un individuo (Lahti, 1999). Otros investigadores también han tomado esta definición precisando - en algunos casos- otros tipos de competencias como los rasgos, motivaciones o intereses (Ismail et al., 2015; Sánchez, 2011, 2013; Schelfhout et al., 2016). Para esta revisión los factores se agrupan en: conocimientos, habilidades, rasgos y otras características.

Tabla 1. Resumen de artículos

\begin{tabular}{|l|l|l|}
\hline \multicolumn{1}{|c|}{ Autor(es) } & \multicolumn{1}{|c|}{\begin{tabular}{c}
\multicolumn{1}{|c|}{ Título del } \\
artículo en español
\end{tabular}} & \multicolumn{1}{c|}{ Resultados } \\
\hline $\begin{array}{l}\text { Ucbasaran, Wes- } \\
\text { thead y Wright } \\
(2009) .\end{array}$ & $\begin{array}{l}\text { El alcance y la naturaleza de la } \\
\text { identificación de oportunida- } \\
\text { des por parte de empresarios } \\
\text { experimentados. }\end{array}$ & $\begin{array}{l}\text { Individuos con emprendimientos previos } \\
\text { identifican más oportunidades. Sin embar- } \\
\text { go, cuando el número de negocios fallidos } \\
\text { es muy alto el número de oportunidades } \\
\text { identificadas cae. }\end{array}$ \\
\hline $\begin{array}{l}\text { Westhead, Ucba- } \\
\text { saran y Wright } \\
(2009) .\end{array}$ & $\begin{array}{l}\text { Búsqueda de información e e } \\
\text { identificación de oportunida- } \\
\text { des: La importancia de la ex- } \\
\text { periencia previa en propiedad } \\
\text { empresarial. }\end{array}$ & $\begin{array}{l}\text { Los emprendedores seriales identifican } \\
\text { más oportunidades que los emprendedores } \\
\text { novatos. } \\
\text { Los emprendedores de portafolio identifi- } \\
\text { can oportunidades que los emprende- } \\
\text { Mayores niveles de capacidad empresarial } \\
\text { mayor intensidad de búsqueda de infor- } \\
\text { mación favorecen a la identificación de } \\
\text { oportunidades. }\end{array}$ \\
\hline
\end{tabular}




\begin{tabular}{|c|c|c|}
\hline $\begin{array}{l}\text { Ramos-Ro- } \\
\text { dríguez, Me- } \\
\text { dina-Garrido, } \\
\text { Lorenzo-Gómez } \\
\text { y Ruiz-Navarro } \\
(2010) \text {. }\end{array}$ & $\begin{array}{l}\text { ¿Qué sabes o a quién conoces? } \\
\text { El papel del capital intelectual } \\
\text { y social en el reconocimiento } \\
\text { de oportunidades. }\end{array}$ & $\begin{array}{l}\text { El acceso al conocimiento externo a través } \\
\text { de las redes sociales en las que participan los } \\
\text { individuos contribuye al desarrollo de capa- } \\
\text { cidades para el reconocimiento de nuevas } \\
\text { oportunidades. }\end{array}$ \\
\hline $\begin{array}{l}\text { Shane, Nicolaou, } \\
\text { Cherkas y Spector } \\
\text { (2010). }\end{array}$ & $\begin{array}{l}\text { ¿La apertura a la experiencia } \\
\text { y el reconocimiento de oportu- } \\
\text { nidades tiene la misma fuente } \\
\text { genética?. }\end{array}$ & $\begin{array}{l}\text { El gen de la apertura a nuevas experiencias } \\
\text { tiene un efecto mediador en el reconoci- } \\
\text { miento de oportunidades. }\end{array}$ \\
\hline $\begin{array}{l}\text { de Bruin y Fe- } \\
\text { rrante (2011). }\end{array}$ & $\begin{array}{l}\text { Oportunidad limitada: un } \\
\text { enfoque basado en el conoci- } \\
\text { miento para el reconocimien- } \\
\text { to y el desarrollo de oportuni- } \\
\text { dades. }\end{array}$ & $\begin{array}{l}\text { El tipo de conocimiento de emprendimien- } \\
\text { to que se posee, ya sea tácito o codificado, } \\
\text { moldea el tipo de oportunidad reconocida. }\end{array}$ \\
\hline $\begin{array}{l}\text { González-Álvarez } \\
\text { y Solís-Rodríguez } \\
\text { (2011). }\end{array}$ & $\begin{array}{l}\text { Descubrimiento de oportu- } \\
\text { nidades de emprendimiento: } \\
\text { Una perspectiva de género. }\end{array}$ & $\begin{array}{l}\text { Los individuos que poseen un mayor stock } \\
\text { de capital humano y tienen redes sociales } \\
\text { más amplias descubren más posibilidades } \\
\text { de creación de negocios. }\end{array}$ \\
\hline $\begin{array}{l}\text { Grégoire y } \\
\text { Shepherd (2012). }\end{array}$ & $\begin{array}{l}\text { Combinaciones de tecnología } \\
\text { y mercado y la identificación } \\
\text { de oportunidades empresaria- } \\
\text { les: una investigación del nexo } \\
\text { oportunidad-individuo. }\end{array}$ & $\begin{array}{l}\text { Las diferencias individuales en cuanto al } \\
\text { conocimiento previo y la intención em- } \\
\text { presarial moderan las relaciones entre las } \\
\text { similitudes superficiales y estructurales de } \\
\text { las oportunidades y la percepción de su } \\
\text { identificación. }\end{array}$ \\
\hline $\begin{array}{l}\text { Li y Gustafsson } \\
(2012) \text {. }\end{array}$ & $\begin{array}{l}\text { Emprendedores nacientes en } \\
\text { China: identidad de clase so- } \\
\text { cial, afiliación previa a la ex- } \\
\text { periencia e identificación de } \\
\text { oportunidades innovadoras. }\end{array}$ & $\begin{array}{l}\text { La identidad de clase social y la experien- } \\
\text { cia previa con otros empresarios nacientes } \\
\text { tienen un impacto significativo en la capa- } \\
\text { cidad de innovación en la etapa de identifi- } \\
\text { cación de oportunidades. }\end{array}$ \\
\hline $\begin{array}{l}\text { Arentz, Sautet y } \\
\text { Storr (2013). }\end{array}$ & $\begin{array}{l}\text { Conocimiento previo e identi- } \\
\text { ficación de oportunidades. }\end{array}$ & $\begin{array}{l}\text { El conocimiento previo no solo influye en } \\
\text { el campo en el que puede tener lugar una } \\
\text { oportunidad, sino también en qué tan aler- } \\
\text { ta puede estar una persona ante las oportu- } \\
\text { nidades en un campo determinado. }\end{array}$ \\
\hline $\begin{array}{l}\text { Wang, Ellinger y } \\
\text { Wu (2013). }\end{array}$ & $\begin{array}{l}\text { Reconocimiento de oportuni- } \\
\text { dades empresariales: un estu- } \\
\text { dio empírico del personal de } \\
\text { I+D. }\end{array}$ & $\begin{array}{l}\text { La autoeficacia individual, el conocimien- } \\
\text { to previo, las redes sociales y la percepción } \\
\text { sobre el entorno industrial sobre las opor- } \\
\text { tunidades tienen efectos positivos en el re- } \\
\text { conocimiento de oportunidades. }\end{array}$ \\
\hline Brockman (2014). & $\begin{array}{l}\text { Alerta emprendedora en la } \\
\text { identificación y desarrollo de } \\
\text { oportunidades. }\end{array}$ & $\begin{array}{l}\text { El conocimiento previo, la experiencia, la } \\
\text { creatividad, el escaneo y la actitud de bús- } \\
\text { queda activa, son factores que influyen en } \\
\text { el estado de alerta empresarial, la cual, a } \\
\text { su vez, afecta el descubrimiento de oportu- } \\
\text { nidades. }\end{array}$ \\
\hline
\end{tabular}




\begin{tabular}{|c|c|c|}
\hline $\begin{array}{l}\text { Chang, Liu y } \\
\text { Chiang (2014). }\end{array}$ & $\begin{array}{l}\text { Un estudio de la relación entre } \\
\text { los cursos de emprendimiento } \\
\text { y la identificación de oportuni- } \\
\text { dades: Una encuesta empírica. }\end{array}$ & $\begin{array}{l}\text { Los cursos de emprendimiento tienen un } \\
\text { impacto significativo en la mejora de las ca- } \\
\text { pacidades de identificación de oportunida- } \\
\text { des y el estado de alerta de emprendimien- } \\
\text { to actúa como variable mediadora de esta } \\
\text { relación. } \\
\text { Los alumnos que poseen conocimientos } \\
\text { previos tienen una mejor oportunidad de } \\
\text { aumentar el estado de alerta empresarial } \\
\text { después de tomar cursos empresariales, } \\
\text { fortaleciendo así su capacidad de identifi- } \\
\text { cación de oportunidades. }\end{array}$ \\
\hline $\begin{array}{l}\text { Gielnik, Krämer, } \\
\text { Kappel y Frese } \\
\text { (2014). }\end{array}$ & $\begin{array}{l}\text { Antecedentes de la identifi- } \\
\text { cación e innovación de opor- } \\
\text { tunidades de negocio: Inves- } \\
\text { tigando la interacción de la } \\
\text { adquisición y el procesamien- } \\
\text { to de la información. }\end{array}$ & $\begin{array}{l}\text { La búsqueda activa de información modera } \\
\text { positivamente el efecto que bajos niveles de } \\
\text { experiencia empresarial tienen en la identi- } \\
\text { ficación de oportunidades, } \\
\text { La búsqueda activa de información mejora } \\
\text { el efecto positivo del pensamiento divergen- } \\
\text { te sobre la identificación de oportunidades. }\end{array}$ \\
\hline $\begin{array}{l}\text { Foo, Uy, \& Mur- } \\
\text { nieks (2015). }\end{array}$ & $\begin{array}{l}\text { Más allá de la Valencia Afecti- } \\
\text { va: Desenredando las influen- } \\
\text { cias de la valencia y la acti- } \\
\text { vación en la identificación de } \\
\text { oportunidades. }\end{array}$ & $\begin{array}{l}\text { Investigaciones futuras se verán beneficia- } \\
\text { das con la inclusión de la valencia afectiva y } \\
\text { la activación afectiva, en las investigaciones } \\
\text { sobre la identificación de oportunidades. }\end{array}$ \\
\hline $\begin{array}{l}\text { Kedmenec, } \\
\text { Šebjan y Tominc } \\
(2015) .\end{array}$ & $\begin{array}{l}\text { Efecto de la Ruralidad y los } \\
\text { Recursos de Capital Humano } \\
\text { en el Proceso de Identificación } \\
\text { de Oportunidades Emprende- } \\
\text { doras. }\end{array}$ & $\begin{array}{l}\text { Altos niveles de fuentes de capital humano, } \\
\text { educación consistente, formación en ha- } \\
\text { bilidades y conocimiento y experiencia en } \\
\text { emprendimiento, tienen un efecto significa- } \\
\text { tivo y positivo en la identificación de opor- } \\
\text { tunidades. }\end{array}$ \\
\hline $\begin{array}{l}\text { Ruiz-Arroyo, } \\
\text { Sanz-Espinosa y } \\
\text { Fuentes-Fuentes } \\
\text { (2015). }\end{array}$ & $\begin{array}{l}\text { Alerta emprendedora y cono- } \\
\text { cimiento previo para la iden- } \\
\text { tificación de oportunidades } \\
\text { emprendedoras: el papel mo- } \\
\text { derador de las redes sociales. }\end{array}$ & $\begin{array}{l}\text { Existe una influencia negativa del conoci- } \\
\text { miento previo sobre el número de oportu- } \\
\text { nidades identificadas. La alerta empren- } \\
\text { dedora conduce a un mayor número de } \\
\text { oportunidades identificadas. }\end{array}$ \\
\hline $\begin{array}{l}\text { Shane y Nicolaou } \\
\text { (2015). }\end{array}$ & $\begin{array}{l}\text { Personalidad creativa, recono- } \\
\text { cimiento de oportunidades y } \\
\text { tendencia a iniciar negocios: } \\
\text { Un estudio de sus predisposi- } \\
\text { ciones genéticas. }\end{array}$ & $\begin{array}{l}\text { Los individuos con personalidades creati- } \\
\text { vas tienen más probabilidades de identifi- } \\
\text { car nuevas oportunidades e iniciar una em- } \\
\text { presa. } \\
\text { Los factores genéticos explican parte de la } \\
\text { correlación entre personalidad creativa y } \\
\text { comportamiento empresarial. }\end{array}$ \\
\hline $\begin{array}{l}\text { Tocher, Oswald y } \\
\text { Hall (2015). }\end{array}$ & $\begin{array}{l}\text { Proponiendo recursos sociales } \\
\text { como catalizadores de la crea- } \\
\text { ción de oportunidades. }\end{array}$ & $\begin{array}{l}\text { El capital social y la competencia social } \\
\text { facilitan la capacidad del empresario para } \\
\text { navegar por el complejo proceso de trans- } \\
\text { formar sus ideas de negocios en oportuni- } \\
\text { dades viables. }\end{array}$ \\
\hline
\end{tabular}




\begin{tabular}{|c|c|c|}
\hline $\begin{array}{l}\text { Barucic y Umiha- } \\
\text { nic (2016). }\end{array}$ & $\begin{array}{l}\text { La educación empresarial } \\
\text { como factor de reconocimien- } \\
\text { to de oportunidades empresa- } \\
\text { riales para iniciar un nuevo } \\
\text { negocio. }\end{array}$ & $\begin{array}{l}\text { El conocimiento y las habilidades de em- } \\
\text { prendimiento están positivamente relacio- } \\
\text { nadas con el reconocimiento de oportuni- } \\
\text { dades. }\end{array}$ \\
\hline $\begin{array}{l}\text { Karimi, Biemans, } \\
\text { Lans, Chizari y } \\
\text { Mulder (2016). }\end{array}$ & $\begin{array}{l}\text { El impacto de la educación } \\
\text { empresarial: un estudio de las } \\
\text { intenciones empresariales y la } \\
\text { identificación de oportunida- } \\
\text { des de estudiantes iraníes. }\end{array}$ & $\begin{array}{l}\text { Los programas electivos y obligatorios de } \\
\text { cursos de educación en emprendimiento } \\
\text { tuvieron impactos positivos y significativos } \\
\text { en las normas subjetivas de los estudiantes, } \\
\text { así como en el control del comportamiento } \\
\text { percibido. } \\
\text { Los programas electivos aumentan signifi- } \\
\text { cativamente la intención empresarial de los } \\
\text { estudiantes, aunque este aumento no es sig- } \\
\text { nificativo para los programas obligatorios. }\end{array}$ \\
\hline $\begin{array}{l}\text { Mueller y } \\
\text { Shepherd } \\
(2016)\end{array}$ & $\begin{array}{l}\text { Aprovechando al máximo las } \\
\text { experiencias de fracaso: Ex- } \\
\text { plorando la relación entre el } \\
\text { fracaso del negocio y la iden- } \\
\text { tificación de oportunidades de } \\
\text { negocio. }\end{array}$ & $\begin{array}{l}\text { El aprendizaje basado en experiencias falli- } \\
\text { das es mayor para aquellos emprendedores } \\
\text { que poseen mayores herramientas cogniti- } \\
\text { vas. } \\
\text { Para los que poseen oportunidades estruc- } \\
\text { turadas y un estilo cognitivo intuitivo, el } \\
\text { conocimiento previo en ámbitos profesio- } \\
\text { nales modera negativamente esta relación. }\end{array}$ \\
\hline $\begin{array}{l}\text { Nikraftar y Hos- } \\
\text { seini } \\
(2016)\end{array}$ & $\begin{array}{l}\text { Factores que afectan al reco- } \\
\text { nocimiento de oportunidades } \\
\text { empresariales en el turismo de } \\
\text { pequeñas y medianas empre- } \\
\text { sas. }\end{array}$ & $\begin{array}{l}\text { La autoeficacia del individuo, el conoci- } \\
\text { miento previo y las redes sociales, tienen } \\
\text { efectos positivos en el estado de alerta em- } \\
\text { presarial. } \\
\text { El estado de alerta empresarial contribu- } \\
\text { ye significativamente al reconocimiento de } \\
\text { oportunidades empresariales. }\end{array}$ \\
\hline $\begin{array}{l}\text { Prandelli, Pas- } \\
\text { quini y Verona } \\
(2016) \text {. }\end{array}$ & $\begin{array}{l}\text { En los zapatos del usuario: } \\
\text { Un diseño experimental sobre } \\
\text { la toma de perspectivas en el } \\
\text { descubrimiento de oportuni- } \\
\text { dades de emprendimiento. }\end{array}$ & $\begin{array}{l}\text { El mecanismo cognitivo de la toma de pers- } \\
\text { pectivas mejora la capacidad de descubrir } \\
\text { oportunidades. } \\
\text { Entender a los clientes y los mercados per- } \\
\text { mite a los emprendedores llenar sus corre- } \\
\text { dores de información con ideas relevantes } \\
\text { y originales. }\end{array}$ \\
\hline $\begin{array}{l}\text { Ren, Shu, Bao y } \\
\text { Chen (2016). }\end{array}$ & $\begin{array}{l}\text { Vinculando los lazos de red } \\
\text { con el descubrimiento y la ex- } \\
\text { plotación de oportunidades } \\
\text { empresariales: el papel de la } \\
\text { confianza afectiva y cognitiva. }\end{array}$ & $\begin{array}{l}\text { Mientras la confianza afectiva media los } \\
\text { efectos positivos de los vínculos de red en } \\
\text { el descubrimiento y explotación de oportu- } \\
\text { nidades, la confianza cognitiva modera sus } \\
\text { relaciones. }\end{array}$ \\
\hline
\end{tabular}




\begin{tabular}{|c|c|c|}
\hline $\begin{array}{l}\text { Song, Min, Lee y } \\
\text { Seo (2017). }\end{array}$ & $\begin{array}{l}\text { Los efectos de la dependencia } \\
\text { de la red en el reconocimiento } \\
\text { de oportunidades: Un modelo } \\
\text { de mediación moderado de } \\
\text { adquisición de conocimiento y } \\
\text { orientación empresarial. }\end{array}$ & $\begin{array}{l}\text { El reconocimiento de oportunidades se lo- } \\
\text { gra más efectivamente a través de la crea- } \\
\text { ción de redes, la adquisición de conoci- } \\
\text { miento y la orientación empresarial. } \\
\text { La adquisición de conocimiento media po- } \\
\text { sitivamente la relación entre la confiabili- } \\
\text { dad de la red y el reconocimiento de opor- } \\
\text { tunidades. }\end{array}$ \\
\hline $\begin{array}{l}\text { St-Jean et al. } \\
(2017) \text {. }\end{array}$ & $\begin{array}{l}\text { ¿Pueden los mentores de nego- } \\
\text { cios actuar como corredores y } \\
\text { habilitadores de oportunida- } \\
\text { des entre los estudiantes uni- } \\
\text { versitarios?. }\end{array}$ & $\begin{array}{l}\text { Los mentores que se encuentran de forma } \\
\text { activa en el mercado mejoran la identifica- } \\
\text { ción y explotación de oportunidades por } \\
\text { parte de sus estudiantes. Por lo tanto, los } \\
\text { mentores actúan como intermediarios y fa- } \\
\text { cilitadores de oportunidades. }\end{array}$ \\
\hline $\begin{array}{l}\text { Hanohov y Bal- } \\
\text { dacchino (2018) }\end{array}$ & $\begin{array}{l}\text { Reconocimiento de oportuni- } \\
\text { dades en el emprendimiento } \\
\text { sostenible: un estudio explora- } \\
\text { torio. }\end{array}$ & $\begin{array}{l}\text { El conocimiento de los entornos naturales } \\
\text { y comunitarios, la motivación para desa- } \\
\text { rrollar ganancias individuales y ganancias } \\
\text { para otros, así como conocimiento de em- } \\
\text { prendimiento, influencian la identificación } \\
\text { de oportunidades sostenibles. }\end{array}$ \\
\hline $\begin{array}{l}\text { Kim, Choi, Sung y } \\
\text { Park (2018) }\end{array}$ & $\begin{array}{l}\text { El papel de la capacidad de } \\
\text { resolución de problemas en el } \\
\text { comportamiento innovador y } \\
\text { el reconocimiento de oportu- } \\
\text { nidades en los estudiantes uni- } \\
\text { versitarios. }\end{array}$ & $\begin{array}{l}\text { A mayor experiencia y conocimientos de } \\
\text { emprendimiento, obtenidos a través de la } \\
\text { resolución de problemas y el comporta- } \\
\text { miento innovador, mayores serán probabi- } \\
\text { lidades de reconocer oportunidades. }\end{array}$ \\
\hline $\begin{array}{l}\text { Schmitt, Rosing, } \\
\text { Zhang y Leather- } \\
\text { bee }(2018)\end{array}$ & $\begin{array}{l}\text { Un modelo dinámico de incer- } \\
\text { tidumbre empresarial e identi- } \\
\text { ficación de oportunidades de } \\
\text { negocio: La exploración como } \\
\text { mediador y la autoeficacia em- } \\
\text { presarial como moderador. }\end{array}$ & $\begin{array}{l}\text { Un aumento en el nivel de incertidumbre } \\
\text { percibida mejora la identificación de opor- } \\
\text { tunidades para emprendedores con alta au- } \\
\text { toeficacia, pero no para aquellos con poca } \\
\text { autoeficacia. }\end{array}$ \\
\hline $\begin{array}{l}\text { Ploum, Blok, Lans } \\
\text { y Omta (2019) }\end{array}$ & $\begin{array}{l}\text { ¿Educar para el interés propio } \\
\text { o la trascendencia? Un enfo- } \\
\text { que empírico para investigar el } \\
\text { papel de las competencias mo- } \\
\text { rales en el reconocimiento de } \\
\text { oportunidades para el desarro- } \\
\text { llo sostenible. }\end{array}$ & $\begin{array}{l}\text { La competencia moral, compuesta por la } \\
\text { competencia normativa y la acción estraté- } \\
\text { gica, tiene una relación significativa con la } \\
\text { cantidad de oportunidades reconocidas. }\end{array}$ \\
\hline
\end{tabular}




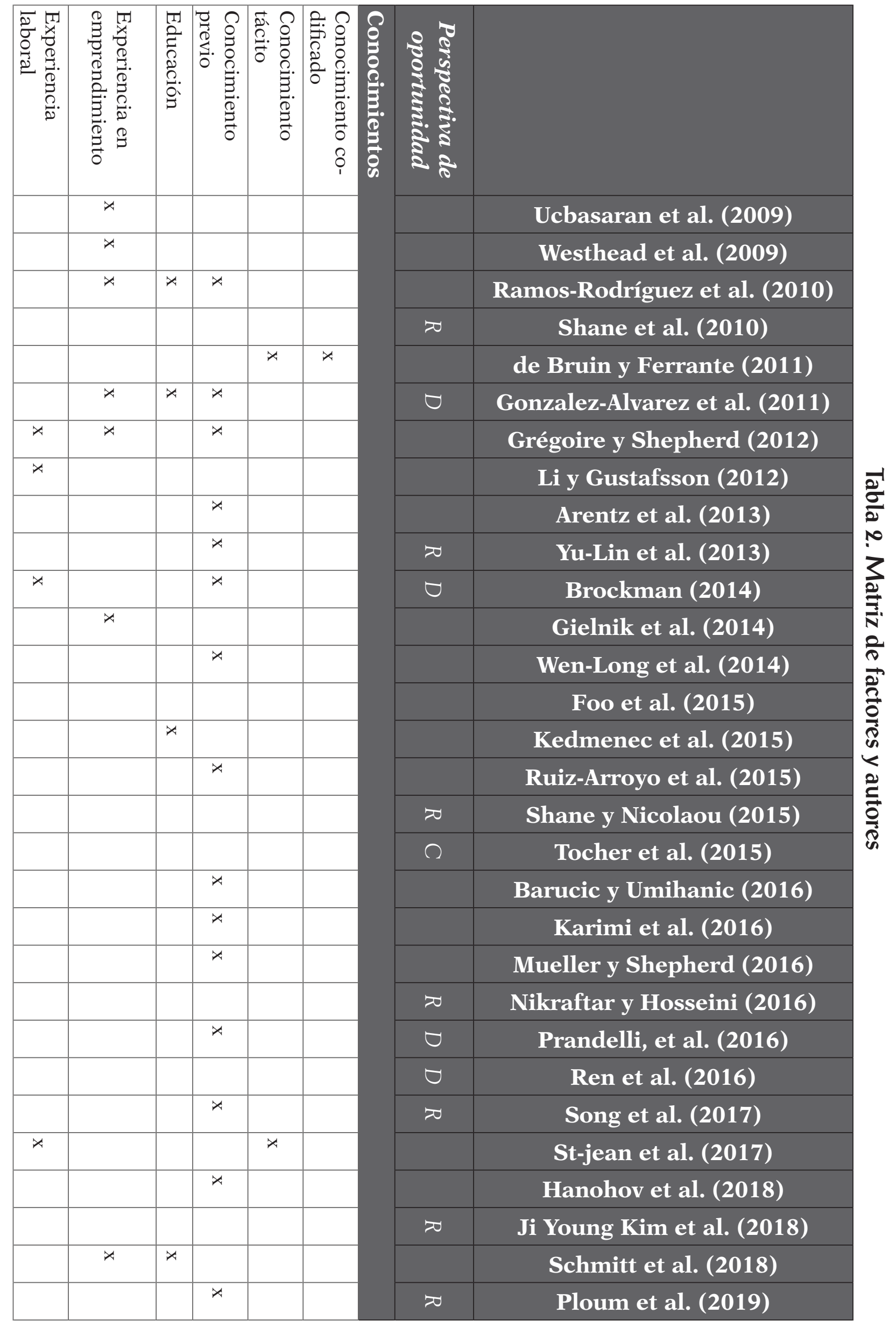




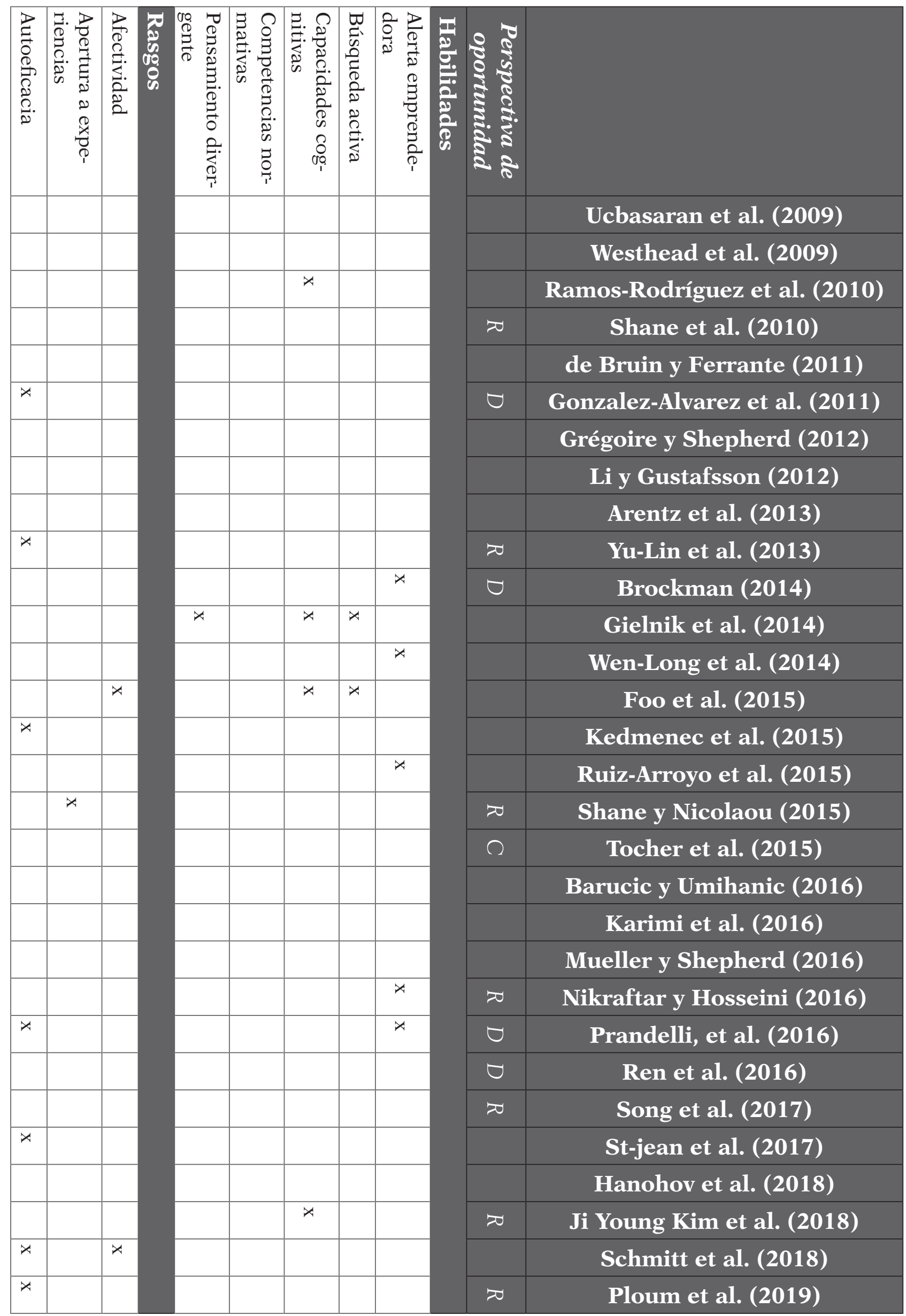




\begin{tabular}{|c|c|c|c|c|c|c|c|}
\hline 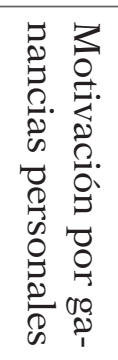 & 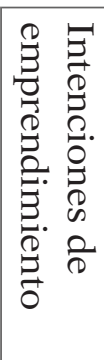 & 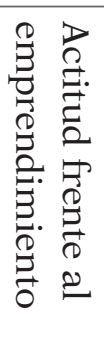 & 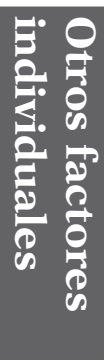 & 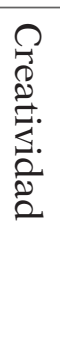 & 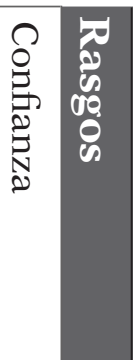 & 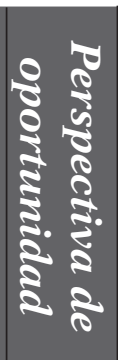 & \\
\hline & & & & & & & Ucbasaran et al. (2009) \\
\hline & & & & & & & Westhead et al. (2009) \\
\hline & & & & & & & Ramos-Rodriguez et al. (2010) \\
\hline & & & & & & 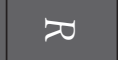 & Shane et al. (2010) \\
\hline & & & & & & & de Bruin y Ferrante (2011) \\
\hline & & & & & & 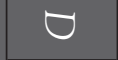 & Gonzalez-Alvarez et al. (2011) \\
\hline & 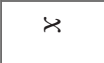 & & & & & & Grégoire y Shepherd (2012) \\
\hline & & & & & & & Li y Gustafsson (2012) \\
\hline & & & & & & & Arentz et al. (2013) \\
\hline & & & & & & 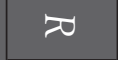 & Yu-Lin et al. (2013) \\
\hline & $x$ & & & $\rtimes$ & & $\nabla$ & Brockman (2014) \\
\hline & & & & & & & Gielnik et al. (2014) \\
\hline & & & & & & & Wen-Long et al. (2014) \\
\hline & & & & & & & Foo et al. (2015) \\
\hline & & & & & & & Kedmenec et al. (2015) \\
\hline & & & & & & & Ruiz-Arroyo et al. (2015) \\
\hline & & & & & & 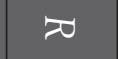 & Shane y Nicolaou (2015) \\
\hline & & & & & & $\Omega$ & Tocher et al. (2015) \\
\hline & & & & & & & Barucic y Umihanic (2016) \\
\hline & & $x$ & & & & & Karimi et al. (2016) \\
\hline & & & & & & & Mueller y Shepherd (2016) \\
\hline & & & & & & $\gg$ & Nikraftar y Hosseini (2016) \\
\hline & $x$ & & & $x$ & & $\nabla$ & Prandelli, et al. (2016) \\
\hline & & & & & $x$ & $\nabla$ & Ren et al. (2016) \\
\hline & & & & & & 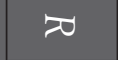 & Song et al. (2017) \\
\hline & & & & & & & St-jean et al. (2017) \\
\hline$x$ & & & & & & & Hanohov et al. (2018) \\
\hline & & & & $x$ & & 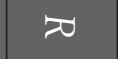 & Ji Young Kim et al. (2018) \\
\hline & & & & $x$ & & & Schmitt et al. (2018) \\
\hline & & & & & & $\gg$ & Ploum et al. (2019) \\
\hline
\end{tabular}




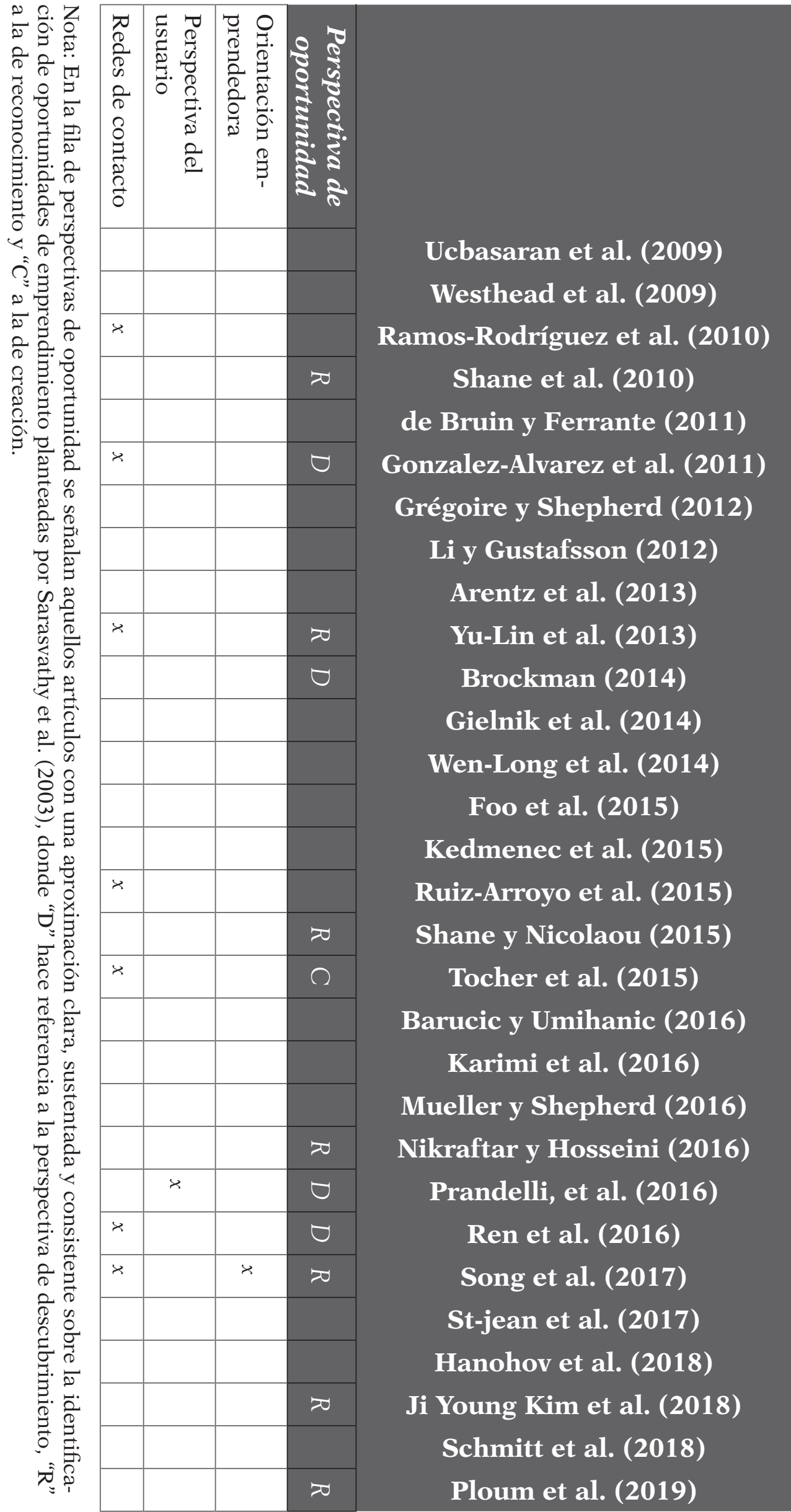




\section{ExperienciaLaboral AlertaEmprendedora MotivacionPorGananciaspersonales

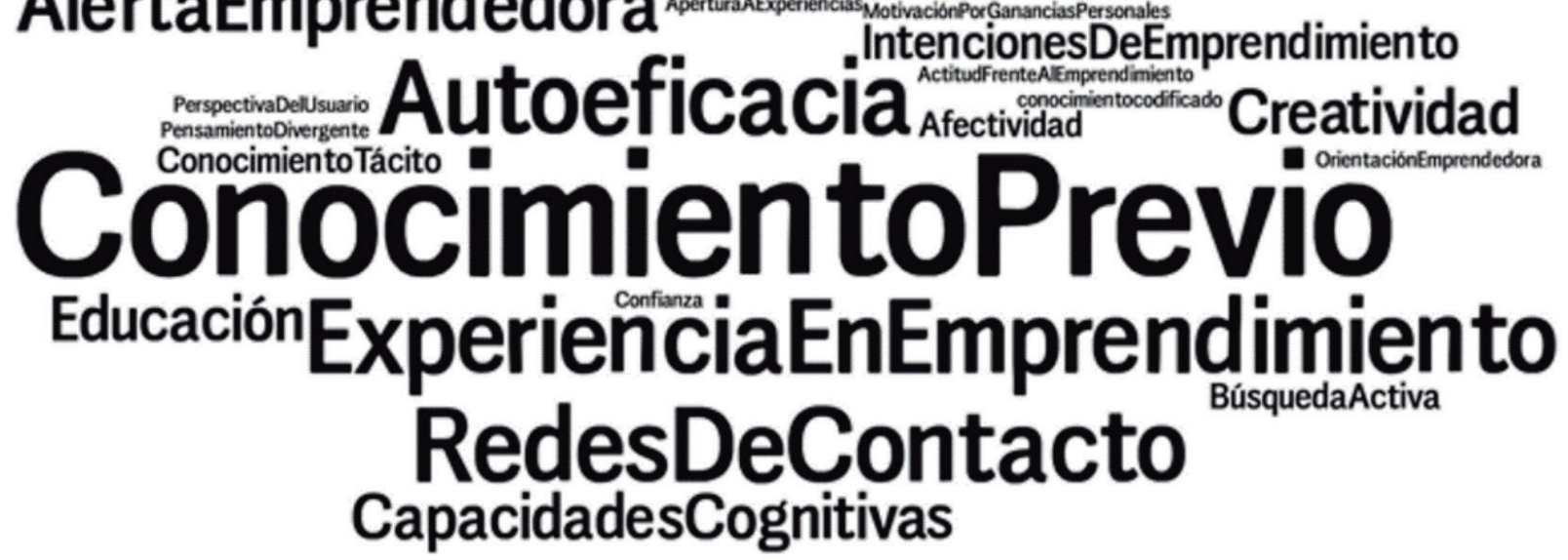

\subsection{Conocimientos}

Esta competencia representa la extensión de la información aprendida por el individuo, que se relaciona directamente con una tarea (Lahti, 1999). En el caso del emprendimiento este conocimiento debe estar relacionado con la información de las ciencias de la administración, las leyes gubernamentales y otros aspectos racionales, tales como la experiencia y la educación (Ismail et al., 2015).

De todos los conceptos dentro esta categoría resalta el del conocimiento previo, el cual comprende todo el acervo de información que posee el individuo, previo al proceso de emprendimiento (Short et al., 2010). El conocimiento previo relevante considera toda aquella información que capacita al individuo para poder identificar, entender, extrapolar, interpretar y aplicar, efectivamente, oportunidades dentro de un mercado específico (Ramos-Rodríguez et al., 2010; Ruiz-Arroyo et al., 2015). Oportunidades que no han podido ser descubiertas por otros individuos y que se pueden transformar en negocios rentables (Gonzalez-Alvarez \& Solis-Rodriguez, 2011).

Las fuentes de donde se pueden adquirir conocimientos relevantes para el emprendimiento se clasifican, generalmente en cuatro categorías. La primera consiste en programas de educación donde se imparten conocimientos relacionados a la administración y al descubrimiento de oportunidades de negocio (Henry et al., 2005b, 2005a). La segunda son experiencias laborales en general, las cuales pueden haber sido desarrolladas en instituciones privadas, gubernamentales o políticas (Li \& Gustafsson, 2012). La tercera son aquellas experiencias de emprendimiento especificas (Corbett, 2005; Driessen \& Zwart, 2006), en las cuales el individuo ha sido propietario o administrador de alguna empresa o ha logrado completar emprendimientos previos (Gielnik et al., 2014). La cuarta son las redes de contacto, las cuales se explicarán más adelante.

\subsection{Habilidades}

Según Lathi (1999), las habilidades son aquellas capacidades que le permiten a un individuo desarrollar tareas intelectuales y físicas, que no requieren el uso de herramientas o equipamiento. Para el caso específico del emprendimiento, estas habi- 
lidades se definen como las capacidades para desarrollar tareas propias de la actividad emprendedora (Ismail et al., 2015).

Una de las habilidades predominantes en el análisis son las capacidades o habilidades cognitivas. Shane y Venkataraman (2000) mencionan que los conocimientos necesarios para emprender (mencionados anteriormente) se obtienen más rápidamente si el emprendedor posee mayores capacidades cognitivas (Shane \& Venkataraman, 2000). Estas capacidades cognitivas permiten a los emprendedores asociar e integrar la información fragmentada que reciben del mercado (Foo et al., 2015) y condicionan las probabilidades de que un individuo descubra oportunidades de emprendimiento especificas en él (Wang et al., 2013). Otras investigaciones han incluido indicadores de las capacidades cognitivas como factores mediadores o moderadores importantes en los procesos de emprendimiento (e.g. Gaglio, 2004; Gielnik et al., 2014; Hartog et al., 2010). Las capacidades cognitivas pueden ser definidas como aquellas habilidades que tiene el individuo, que le permiten descomponer y entender la información que este recibe de las diferentes fuentes antes descritas, obteniendo de ellas conclusiones relevantes que le permiten resolver problemas reales de emprendimiento (Gielnik et al., 2014).

Otra habilidad que potencia el conocimiento para emprendimiento es la búsqueda activa, la cual involucra esfuerzos consientes de encontrar y acumular información relevante, relacionada a las nuevas oportunidades que va identificado o desea identificar (Foo et al., 2015). Otras dos habilidades, con menos presencia en nuestro análisis, son: a) las competencias normativas, las cuales son especialmente importantes para reconocer oportunidades relacionadas al desarrollo sostenible, como lo menciona Ploum et al. (2019); y b) el pensamiento divergente, el cual está relacionado a la creatividad y le permite al individuo generar una mayor cantidad de ideas, más originales y de mayor relevancia para el emprendimiento (Gielnik et al., 2014).

Por último, el estado de alerta emprendedora ha sido, también, ampliamente incluido en investigaciones relacionadas a la identificación de oportunidades. Este factor se define como la habilidad que tiene un individuo para percibir oportunidades, ignoradas por otros, sin necesidad de buscarlas intencionalmente (Chang et al., 2014; Kirzner, 1973). En otras palabras, es la sensibilidad del individuo a la información sobre objetos, incidentes y patrones de comportamiento en su entorno, especialmente, a los problemas de los fabricantes y de los usuarios, necesidades e intereses no satisfechos y nuevas combinaciones de recursos (Ray \& Cardozo, 1996, en Ardichvili et al., 2003, p. 113).

\subsection{Rasgos}

Los rasgos de la personalidad, son características duraderas y predecibles de los individuos, que ayudan a explicar su comportamiento y el por qué estos actúan de diferentes maneras en situaciones similares (Hwee Nga \& Shamuganathan, 2010). Estos elementos también ayudan a predecir la propensión a actuar que tiene un individuo (McCrae \& Costa, 1990, en Rauch \& Frese, 2007). Más específicamente, los rasgos relacionados al emprendimiento son aquellos que incentivan o incrementan la propensión de un individuo a emprender.

En términos generales, teóricos contemporáneos coinciden en que existen cinco grandes rasgos de la personalidad, explicados por el modelo conocido como "Big five" o modelo de los cinco factores (McCrae \& Costa, 1997). Este modelo, cuyos factores 
son: a) extraversión; b) neuroticismo; c) afabilidad, d) escrupulosidad; y e) apertura a experiencias, ha sido ampliamente utilizado y aplicado por múltiples estudios sobre emprendimiento (e.g. Brandstätter, 2011; Obschonka et al., 2012; Zhao et al., 2010). Por ejemplo, la apertura a experiencias describe el grado en el cual el individuo es imaginativo, curioso, original y está abierto a nuevas ideas (Shane et al., 2010).

Sin embargo, los artículos de esta revisión que incluyen rasgos de la personalidad, se han enfocado en rasgos más específicos, los cuales permiten identificar características puntuales de quienes logran llevar a cabo el proceso de emprendimiento con éxito (Jain, 2011; Martin et al., 2013; Miao et al., 2017; Rauch \& Frese, 2007; Unger et al., 2011). La autoeficacia es el rasgo más predominantemente investigado y se define como la creencia que posee un individuo en su capacidad de reunir e implementar los recursos personales, habilidades y competencias necesarias para alcanzar un cierto nivel de logro en una tarea determinada (Bandura, 1977). Este concepto está cercanamente relacionado con el nivel de confianza que posee el individuo (Ren et al., 2016), otro factor encontrado en nuestra revisión.

Otro rasgo encontrado es la creatividad y se define como la "habilidad del individuo de descubrir algo que es nuevo, útil y valioso” (Jain, 2011, p. 132). En su versión unidimensional, la creatividad ha sido un constructo bastante relacionado con el emprendimiento, especialmente para el enfoque de creación de oportunidades (Gielnik et al., 2012; Wright et al., 2007).

\subsection{Otros factores individuales}

Otro de los factores predominantes son las redes de contacto que posee la persona. Especialmente importante para la perspectiva de identificación de oportunidades propuesta por Kirzner (1973), las redes de contacto son fuentes de información crucial, ya que permiten el acceso a conocimiento potencialmente valioso (DeTienne \& Chandler, 2004; Sarasvathy et al., 2003). Según Song et al. (2017) las redes de contacto pueden ser definidas como la suma de conexiones que el individuo posee con otras personas u organizaciones de su entorno, las cuales tienen la finalidad de intercambiar información relevante sobre el mercado.

Este factor, además, está cercanamente relacionado a los corredores de información mencionados por Shane (2000), quien sostiene que dichos corredores entregan mayores posibilidades de obtener información valiosa sobre los clientes, cambios en el mercado y demás información importante (Ardichvili et al., 2003; Eckhardt \& Shane, 2010; Lehner \& Kaniskas, 2012). Además, las redes de contacto representan una orientación social por parte del emprendedor (Abuzhuri \& Hashim, 2017) una vez que este ha comprendido que para poder concretar ideas de negocio, se requiere de conexiones con otros individuos (Oosterbeek et al., 2010, p. 446). En los artículos revisados, también se encontraron algunas investigaciones (Gonzalez-Alvarez \& SolisRodriguez, 2011; Ramos-Rodríguez et al., 2010; Tocher et al., 2015) donde se utiliza el término capital social, para hacer referencia a las redes de contacto y las normas de reciprocidad asociadas a ellas. Más específicamente, Song et al. (2017) utiliza, en su lugar, el factor "confiabilidad de la red de contactos", la cual no solo evalúa el tamaño de la red sino también su calidad.

Por otro lado, las intenciones de emprendimiento (elemento que se ha incluido en varias de las investigaciones analizadas) representan el grado en el cual un individuo 
considera iniciar una empresa en el futuro cercano (Grégoire \& Shepherd, 2012), es decir, su propensión a convertirse en emprendedor (Prandelli et al., 2016). Según la teoría del comportamiento planificado de Ajzen (1991)1985, 1987, las intenciones de realizar una determinada actividad son un predictor efectivo de la actividad real, por lo cual es ideal para medir acciones que suceden en momentos inciertos o muy difíciles de encontrar, como lo es el inicio de un emprendimiento.

Los últimos tres factores encontrados en esta revisión, han tenido presencia en algunos pocos artículos. El primero, las motivaciones por ganancias personales, son incluidos en el artículo de Hanohov y Baldacchino (2018), quienes sustentan que este tipo de motivación incrementa la atención que ofrece el individuo hacia oportunidades viables y sostenibles en el tiempo, por lo cual se incrementan también, sus probabilidades de reconocer una oportunidad de emprendimiento sostenible. Por su lado, la orientación emprendedora otorga al individuo una mayor propensión a tomar riesgos, mayor innovación y proactividad, las cuales proveen de una actitud más adecuada para la toma de decisiones de emprendimiento, como lo es la identificación de oportunidades (Song et al., 2017). Finalmente, la perspectiva de usuario es incluida en la investigación de Prandelli et al. (2016) como un elemento importante para la identificación de oportunidades de emprendimiento ya que generan, en el emprendedor, habilidades le permiten interpretar estados mentales y anticipar acciones de potenciales consumidores.

\section{Conclusiones}

De todos los factores individuales encontrados en el análisis, resaltan cinco, por tener una mayor presencia en los artículos y por presentar efectos positivos y significativos en la identificación (o creación) de oportunidades para emprendimiento: el conocimiento previo, el estado de alerta emprendedora, la autoeficacia, la creatividad, y las redes de contacto. El conocimiento previo permite que los individuos tengan mayores herramientas para identificar o desarrollar oportunidades rentables, tal como se menciona en la célebre frase de Louis Pasteur: la fortuna favorece a las mentes preparadas. La alerta emprendedora le permite a un individuo identificar oportunidades de negocio, que otros ignoran, sin la necesidad de buscarlas intencionalmente. La autoeficacia es un factor que caracteriza a los emprendedores que confían en las capacidades que ha adquirido durante su vida, lo cual le ofrece mayor determinación al momento de definir si ha encontrado o no una oportunidad de negocio real. Por su parte, la creatividad es un rasgo de la personalidad que es de suma importancia para todo tipo de emprendimientos, pero especialmente para aquellas ideas de negocio que se basan en la creación de oportunidades de negocio. Finalmente, las redes de contacto son un potenciador importante para otros factores, sobre todo para la obtención de información y conocimientos, los cuales sirven, finalmente, para conectar ideas e identificar así, una mayor cantidad de oportunidades para el emprendimiento.

\section{Limitaciones e investigaciones futuras}

Esta investigación posee limitaciones. La primera es que el análisis de los artículos revisados fueron obtenidos de los repositorios de ABI/Inform, Emerald y Web of Science, los cuales incluyen un grupo importante de investigaciones sobre oportuni- 
dades de emprendimiento (George et al., 2016). Sin embargo, investigaciones futuras pueden realizarse incluyendo la base de datos SciVerse de SCOPUS, la cual es la base de datos de artículos relacionados a emprendimiento, más completa en la actualidad.

La segunda limitación, presente en todo artículo de revisión, es que se podrían estar excluyendo artículos relevantes, que no han sido correctamente catalogados, o que no cuenten con los términos clave utilizados para la búsqueda, en sus títulos, resúmenes u otros metadatos importantes. Por otro lado, si bien esta revisión incluye tanto artículos publicados en idioma inglés, como en español, un número no despreciable de artículos han sido publicados en otros idiomas, diferentes a los mencionados. Por ello, investigaciones futuras se beneficiarían por la inclusión de investigaciones realizadas en otros idiomas o, en su defecto, de la traducción de artículos en diferentes idiomas.

Futuras investigaciones son necesarias, también, para poder reforzar los hallazgos y conclusiones a las que se han llegado en esta revisión. Además, sería importante que revisiones de literatura futuras se enfoquen, también, en las demás etapas del emprendimiento. Es decir, que puedan analizar los factores relevantes para la evaluación y explotación de oportunidades de emprendimiento. Una implicancia para los investigadores es que los hallazgos de esta investigación promueven la búsqueda de brechas de investigación, los cuales pueden ser analizados en estudios posteriores.

\section{Referencias}

Abuzhuri, N.M., \& Hashim, N.B. (2017). The Role of Entrepreneurial Opportunity Recognition on Relationship among Entrepreneurship Education and Entrepreneurial Career Option. European Journal of Business and Management, 9(30), 99-106.

Ajzen, I. (1991). The theory of planned behavior. Orgnizational Behavior and Human Decision Processes, 50, 179-211. https://doi.org/10.1016/0749-5978(91)90020-T

Ardichvili, A., Cardozo, R., \& Ray, S. (2003). A Theory of Entrepreneurial Opportunity Identification and Development. Journal of Business Venturing, 18, 105-123. https://doi.org/10.1016/S0883-9026(01)00068-4

Arentz, J., Sautet, F., \& Storr, V. (2013). Prior-knowledge and opportunity identification. Small Business Economics, 41(2), 461-478. https://doi.org/10.1007/s11187-012-9437-9

Bae, T. J., Qian, S., Miao, C., \& Fiet, J.O. (2014). The Relationship Between Entrepreneurship Education and Entrepreneurial Intentions: A Meta-Analytic Review. Entrepreneurship: Theory and Practice, 38(2), 217-254. https://doi.org/10.1111/etap.12095

Bandura, A. (1977). Self-efficacy: Toward a unifying theory of behavioral change. Psychological Review, 84(2), 191-215. https://doi.org/10.1037/0033-295X.84.2.191

Baron, R. a. (2006). Opportunity Recognition as Pattern Recognition. Academy of Management Perspectives, 20, 104-120. https://doi.org/10.5465/AMP.2006.19873412

Barucic, A., \& Umihanic, B. (2016). Entrepreneurship Education as a Factor of Entrepreneurial Opportunity Recognition for Starting a New Business. Management, 21(2), 27-44.

Brandstätter, H. (2011). Personality aspects of entrepreneurship: A look at five meta-analyses. Personality and Individual Differences, 51(3), 222-230. https://doi.org/10.1016/j.paid.2010.07.007

Brockman, B.K. (2014). Entrepreneurial Alertness in Opportunity Identification and Opportunity Development. Journal of Business and Entrepreneurship, 26(2), 27-44. https://bit.ly/31 if1 mC

Chang, W.L., Liu, W.G.H., \& Chiang, S.M. (2014). A study of the relationship between entrepreneurship courses and opportunity identification: An empirical survey. Asia Pacific Management Review, 19(1), 1-24. https://doi.org/10.6126/APMR.2014.19.1.01

Corbett, A.C. (2005). Experiential learning within the process of opportunity identification and exploitation. Entrepreneurship Theory and Practice, 29(4), 473-491.

https://doi.org/10.1111/j.1540-6520.2005.00094.x 
de Bruin, A.M., \& Ferrante, F.M. (2011). Bounded Opportunity: A Knowledge-Based Approach to Opportunity Recognition and Development. Entrepreneurship Research Journal, 1(4). https://doi.org/10.2202/2157-5665.1018

DeTienne, D.R., \& Chandler, G.N. (2004). Opportunity Identification and Its Role in the Entrepreneurial Classroom: A Pedagogical Approach and Empirical Test. Academy of Management Learning $\mathcal{E}$ Education, 3(3), 242-257. https://doi.org/10.5465/AMLE.2004.14242103

Driessen, M.P., \& Zwart, P.S. (2006). De E-Scan Ondernemersdtest ter beoordeling van ondernemerscharp. 4(4), 14. https://bit.ly/33ql37e

Eckhardt, J. T., \& Shane, S. (2003). Opportunities and entrepreneurship. Journal of Management, 29(3), 333-349. https://doi.org/10.1016/S0149-2063(02)00225-8

Eckhardt, J. T., \& Shane, S. (2010). Handbook of Entrepreneurship Research. Handbook of Entrepreneurship Research (pp. 47-76). https://doi.org/10.1007/978-1-4419-1191-9

Fayezi, S., Zutshi, A., \& O'Loughlin, A. (2017). Understanding and Development of Supply Chain Agility and Flexibility: A Structured Literature Review. International Journal of Management Reviews, 19(4), 379-407. https://doi.org/10.1111/ijmr.12096

Foo, M. Der, Uy, M.A., \& Murnieks, C. (2015). Beyond Affective Valence: Untangling Valence and Activation Influences on Opportunity Identification. Entrepreneurship: Theory and Practice, 39(2), 407-431. https://doi.org/10.1111/etap.12045

Gaglio, C.M. (2004). The role of mental simulations and counterfactual thinking in the opportunity identification process. Entrepreneurship-Theory and Practice, 28(6), 533-552.

https://doi.org/10.1111/j.1540-6520.2004.00063.x

Gartner, W.B., Shaver, K.G., \& Liao, J.J. (2008). Opportunities As Attributions: Categorizing Strategic Issues From An Attributional Perspective. Strategic Entrepreneurship Journal, 2(1), 301-315. https://doi.org/10.1002/sej

George, N.M., Parida, V., Lahti, T., \& Wincent, J. (2016). A systematic literature review of entrepreneurial opportunity recognition: insights on influencing factors. International Entrepreneurship and Management Journal, 12(2), 309-350. https://doi.org/10.1007/s11365-014-0347-y

Gielnik, M.M., Frese, M., Graf, J.M., \& Kampschulte, A. (2012). Creativity in the opportunity identification process and the moderating effect of diversity of information. Journal of Business Venturing, 27(5), 559-576. https://doi.org/10.1016/j.jbusvent.2011.10.003

Gielnik, M.M., Krämer, A.C., Kappel, B., \& Frese, M. (2014). Antecedents of Business Opportunity Identification and Innovation: Investigating the Interplay of Information Processing and Information Acquisition. Applied Psychology, 63(2), 344-381. https://doi.org/10.1111/j.1464-0597.2012.00528.x

Gonzalez-Alvarez, N., \& Solis-Rodriguez, V. (2011). Discovery of entrepreneurial opportunities: A gender perspective. Industrial Management and Data Systems, 111(5), 755-775. https://doi.org/10.1108/02635571111137296

Grégoire, D.A., Corbett, A.C., \& Mcmullen, J.S. (2011). The Cognitive Perspective in Entrepreneurship : An Agenda for Future Research The Cognitive Perspective in Entrepreneurship : An Agenda for Future Research. Journal of Management Studies, 6(48), 1443-1477. https://doi.org/10.1111/j.1467-6486.2010.00922.x

Grégoire, D.A., \& Shepherd, D.A. (2012). Technology-market combinations and the identification of entrepreneurial opportunities: An investigation of the opportunity-individual nexus. Academy of Management Journal, 55(4), 753-785. https://doi.org/10.5465/amj.2011.0126

Hanohov, R., \& Baldacchino, L. (2018). Opportunity recognition in sustainable entrepreneurship: an exploratory study. International Journal of Entrepreneurial Behaviour and Research, 24(2), 333-358. https://doi.org/10.1108/IJEBR-12-2015-0275

Hartog, J., Van Praag, M., \& Van Der Sluis, J. (2010). If You Are So Smart, Why Aren't You an Entrepreneur? Returns to Cognitive and Social Ability: Entrepreneurs Versus Employees. Journal of Economics and Management Strategy, 19(4), 947-989.

https://doi.org/10.1111/j.1530-9134.2010.00274.x 
Henry, C., Hill, F., \& Leitch, C. (2005a). Entrepreneurship education and training: can entrepreneurship be taught? Part I. Education + Training, 47(2), 98-111. https://doi.org/10.1108/00400910510586524

Henry, C., Hill, F., \& Leitch, C. (2005b). Entrepreneurship education and training: can entrepreneurship be taught? Part II. Education + Training, 47(3), 158-169. https://doi.org/10.1108/00400910510592211

Hmieleski, K.M., \& Powell, E.E. (2017). The Psychological Foundations of University Science Commercialization: A Review of the Literature and Directions for Future Research. The Academy of Management Perspectives, 32(1), 43-77. https://doi.org/10.5465/amp.2016.0139

Hwee Nga, J.K., \& Shamuganathan, G. (2010). The influence of personality traits and demographic factors on social entrepreneurship start up intentions. Journal of Business Ethics, 95(2), 259282. https://doi.org/10.1007/s10551-009-0358-8

Ismail, V.Y., Zain, E., \& Zulihar. (2015). The Portrait of Entrepreneurial Competence on Student Entrepreneurs. Procedia - Social and Behavioral Sciences, 169(August 2014), 178-188. https://doi. org/10.1016/j.sbspro.2015.01.300

Jain, R.K. (2011). Entrepreneurial Competencies: A Meta-analysis and Comprehensive Conceptualization for Future Research. Vision: The Journal of Business Perspective, 15(2), 127-152. https://doi. org/10.1177/097226291101500205

Karimi, S., Biemans, H.J.A., Lans, T., Chizari, M., \& Mulder, M. (2016). The Impact of Entrepreneurship Education: A Study of Iranian Students' Entrepreneurial Intentions and Opportunity Identification. Journal of Small Business Management, 54(1), 187-209. https://doi.org/10.1111/jsbm.12137

Kedmenec, I., Šebjan, U., \& Tominc, P. (2015). Effect of Rurality and Human Capital Resources in the Entrepreneurial Opportunity Identification Process / Vpliv ruralnega okolja in človeškega kapitala v procesu zaznavanja podjetniških priložnosti. Naše Gospodarstvo/Our Economy, 61(2), 35-44. https://doi.org/10.1515/ngoe-2015-0008

Kim, J.Y., Choi, D.S., Sung, C.S., \& Park, J.Y. (2018). The role of problem solving ability on innovative behavior and opportunity recognition in university students. Journal of Open Innovation: Technology, Market, and Complexity, 4(1). https://doi.org/10.1186/s40852-018-0085-4

Kirzner, I. M. (1973). Competition and Entrepreneurship. University of Chicago Press.

Lahti, R. K. (1999). Identifying and Integrating Individual Level and Organizational Level Core Competencies. Journal of Business and Psychology, 14(1), 59-75. https://doi.org/10.1023/A:1022906400927

Lehner, O.M., \& Kaniskas, J. (2012). Opportunity recognition in social entrepreneurship: A thematic meta analysis. Journal of Entrepreneurship, 21(1), 25-58. https://doi.org/10.1177/097135571102100102

Li, T., \& Gustafsson, V. (2012). Nascent entrepreneurs in China: social class identity, prior experience affiliation and identification of innovative opportunity. Chinese Management Studies, 6(1), 14-35. https://doi.org/10.1108/17506141211213708

Martin, B.C., McNally, J.J., \& Kay, M.J. (2013). Examining the formation of human capital in entrepreneurship: A meta-analysis of entrepreneurship education outcomes. Journal of Business Venturing, 28(2), 211-224. https://doi.org/10.1016/j.jbusvent.2012.03.002

McCrae, R.R., \& Costa, P.T. (1997). Personality Trait Structure as a Human Universal. American Psychologist, 52(5), 509-516.

Miao, C., Qian, S., \& Ma, D. (2017). The Relationship between Entrepreneurial Self-Efficacy and Firm Performance: A Meta-Analysis of Main and Moderator Effects. Journal of Small Business Management, 55(1), 87-107. https://doi.org/10.1111/jsbm.12240

Mitchelmore, S., \& Rowley, J. (2013). Entrepreneurial competencies of women entrepreneurs pursuing business growth. Journal of Small Business and Enterprise Development, 20(1), 125-142. https:// doi.org/10.1108/14626001311298448

Mueller, B. A., \& Shepherd, D. A. (2016). Making the Most of Failure Experiences: Exploring the Relationship Between Business Failure and the Identification of Business Opportunities. 
Entrepreneurship: Theory and Practice, 40(3), 457-487. https://doi.org/10.1111/etap.12116

Nikraftar, T., \& Hosseini, E. (2016). Factors affecting entrepreneurial opportunities recognition in tourism small and medium sized enterprises. Tourism Review, 71(1), 6-17.

https://doi.org/10.1108/TR-09-2015-0042

Obschonka, M., Silbereisen, R.K., \& Schmitt-Rodermund, E. (2012). Explaining Entrepreneurial Behavior : Dispositional Personality ... The Career Development Quarterly, 60(June), 178-191. https://doi.org/10.1002/j.2161-0045.2012.00015.x

Oosterbeek, H., van Praag, M., \& Ijsselstein, A. (2010). The impact of entrepreneurship education on entrepreneurship skills and motivation. European Economic Review, 54(3), 442-454. https:// doi.org/10.1016/j.euroecorev.2009.08.002

Platt, J. (1981). Evidence and proof in documentary research: 1. Some specific problems of documentary research. Sociological Review, 29(1), 31-52. https://bit.ly/2DBjHvo

Ploum, L., Blok, V., Lans, T., \& Omta, O. (2019). Educating for self-interest or -transcendence? An empirical approach to investigating the role of moral competencies in opportunity recognition for sustainable development. Business Ethics, 28(2), 243-260.

https://doi.org/10.1111/beer.12214

Prandelli, E., Pasquini, M., \& Verona, G. (2016). In user's shoes: An experimental design on the role of perspective taking in discovering entrepreneurial opportunities. Journal of Business Venturing, 31(3), 287-301. https://doi.org/10.1016/j.jbusvent.2016.02.001

Ramos-Rodríguez, A. R., Medina-Garrido, J.A., Lorenzo-Gómez, J.D., \& Ruiz-Navarro, J. (2010). What you know or who you know? The role of intellectual and social capital in opportunity recognition. International Small Business Journal, 28(6), 566-582. https://doi.org/10.1177/0266242610369753

Rauch, A., \& Frese, M. (2007). Let's put the person back into entrepreneurship research: A meta-analysis on the relationship between business owners' personality traits, business creation, and success. European Journal of Work and Organizational Psychology, 16(4), 353-385. https:// doi.org/10.1080/13594320701595438

Ren, S., Shu, R., Bao, Y., \& Chen, X. (2016). Linking network ties to entrepreneurial opportunity discovery and exploitation: the role of affective and cognitive trust. International Entrepreneurship and Management Journal, 12(2), 465-485. https://doi.org/10.1007/s11365-014-0350-3

Ruiz-Arroyo, M., Sanz-Espinosa, I., \& Fuentes-Fuentes, M. del M. (2015). Alerta emprendedora y conocimiento previo para la identificación de oportunidades emprendedoras: El papel moderador de las redes sociales. Investigaciones Europeas de Dirección y Economía de la Empresa, 21(1), 47-54. https://doi.org/10.1016/j.iedee.2014.07.002

Sánchez-Meca, J. (2010). Cómo realizar una revisión sistemática y un meta-análisis. Aula Abierta, 38(2), 53-63.

Sánchez, J.C. (2011). University training for entrepreneurial competencies: Its impact on intention of venture creation. International Entrepreneurship and Management Journal, 7(2), 239-254. https:// doi.org/10.1007/s11365-010-0156-x

Sánchez, J.C. (2013). The impact of an entrepreneurship education program on entrepreneurial competencies and intention. Journal of Small Business Management, 51(3), 447-465. https://doi.org/10.1111/jsbm.12025

Sarasvathy, S. D., Dew, N., Velamuri, S. R., \& Venkataraman, S. (2003). Three Views of Entrepreneurial Opportunity. In Springer (Ed.), Handbook of Entrepreneurship Research (pp. 141-160). https://doi.org/10.1007/978-1-4419-1191-9_4

Schelfhout, W., Bruggeman, K., \& De Mayer, S. (2016). Evaluation of entrepreneurial competence through scaled behavioural indicators: Validation of an instrument. Studies in Educational Evaluation, 51, 29-41. https://doi.org/10.1016/j.stueduc.2016.09.001

Schmitt, A., Rosing, K., Zhang, S.X., \& Leatherbee, M. (2018). A Dynamic Model of Entrepreneurial Uncertainty and Business Opportunity Identification: Exploration as a Mediator and Entrepreneurial Self-Efficacy as a Moderator. In Entrepreneurship Theory and Practice (Vol. 42, Issue 6). https://doi.org/10.1177/1042258717721482 
Schumpeter, J. A. (1934). The theory of economic development. Harvard University Press.

Shane, S. (2012). Reflections On The 2010 Amr Decade Award: Delivering On The Promise Of Entrepreneurship As A Field Of Research. Academy of Management Review, 37(January), 10-20. https://doi.org/10.5465/armr.2011.0079

Shane, S., \& Nicolaou, N. (2015). Creative personality, opportunity recognition and the tendency to start businesses: A study of their genetic predispositions. Journal of Business Venturing, 30(3), 407-419. https://doi.org/10.1016/j.jbusvent.2014.04.001

Shane, S., Nicolaou, N., Cherkas, L., \& Spector, T. D. (2010). Do Openness To Experience And Recognizing Opportunities Have The Same Genetic Source? Human Resource Management, 49(2), 291- 303. https://doi.org/10.1002/hrm

Shane, S., \& Venkataraman, S. (2000). The Promise of Entrepreneurship as a Field of Research. The Academy of Management Review, 25(1), 217-226. https://doi.org/10.2307/259271

Short, J.C., Ketchen, D.J., Shook, C.L., \& Ireland, R.D. (2010). The concept of "Opportunity" in entrepreneurship research: Past accomplishments and future challenges. Journal of Management, 36(1), 40-65. https://doi.org/10.1177/0149206309342746

Song, G., Min, S., Lee, S., \& Seo, Y. (2017). The effects of network reliance on opportunity recognition: A moderated mediation model of knowledge acquisition and entrepreneurial orientation. Technological Forecasting and Social Change, 117, 98-107. https://doi.org/10.1016/j.techfore.2017.01.004

St-Jean, É., Tremblay, M., Janssen, F., Baronet, J., Loué, C., \& Nafa, A. (2017). May business mentors act as opportunity brokers and enablers among university students? International Entrepreneurship and Management Journal, 13(1), 97-111. https://doi.org/10.1007/s11365-016-0397-4

Tocher, N., Oswald, S.L., \& Hall, D.J. (2015). Proposing social resources as the fundamental catalyst toward opportunity creation. Strategic Entrepreneurship Journal, 9(2), 119-135. https://doi.org/10.1002/sej.1195

Ucbasaran, D., Westhead, P., \& Wright, M. (2009). The extend and nature of opportunity identification by experienced entrepreneurs. Journal of Business Venturing, 24(1), 99-115.

Unger, J. M., Rauch, A., Frese, M., \& Rosenbusch, N. (2011). Human capital and entrepreneurial success: A meta-analytical review. Journal of Business Venturing, 26(3), 341-358. https://doi.org/10.1016/j.jbusvent.2009.09.004

Venkataraman, S. (1997). The Distinctive Domain of Entrepreneurship Research. Advances in Entrepreneurship, Firm Emergence and Growth, 3(October), 119-138. https://doi.org/10.2139/ssrn.1444184

Wang, Y.L., Ellinger, A.D., \& Wu, Y.C. J. (2013). Entrepreneurial opportunity recognition: An empirical study of R\&D personnel. Management Decision, 51(2), 248-266. https://doi.org/10.1108/00251741311301803

Webster, J., \& Watson, R.T. (2011). Analyzing the Past To Prepare for the Future: Writing a Review. MIS Quartely, 26(2), 12. www.jstor.org/stable/4132319

Westhead, P., Ucbasaran, D., \& Wright, M. (2009). Information Search and Opportunity Identification: The Importance of prior Business Ownership Experience. International Small Business Journal, 27(6), 659-680. https://doi.org/10.1177/0266242609344255

Wright, M., Hmieleski, K.M., Siegel, D.S., \& Ensley, M.D. (2007). The Role of Human Capital in Technological Entrepreneurship. Entrepreneurship Theory and Practice, 31(6), 791-806. https://bit.ly/3hLz8AB

Zhao, H., Seibert, S.E., \& Lumpkin, G.T. (2010). The relationship of personality to entrepreneurial intentions and performance: A meta-analytic review. Journal of Management, 36(2), 381-404. https://doi.org/10.1177/0149206309335187 